\title{
Dynamic Euler-Bernoulli Beam Equation: Classification and Reductions
}

\author{
R. Naz ${ }^{1}$ and F. M. Mahomed ${ }^{2}$ \\ ${ }^{1}$ Centre for Mathematics and Statistical Sciences, Lahore School of Economics, Lahore 53200, Pakistan \\ ${ }^{2}$ DST-NRF Centre of Excellence in Mathematical and Statistical Sciences, School of Computer Science and Applied Mathematics, \\ University of the Witwatersrand, Johannesburg, Wits 2050, South Africa \\ Correspondence should be addressed to R. Naz; drrehana@lahoreschool.edu.pk
}

Received 8 May 2015; Revised 20 August 2015; Accepted 23 August 2015

Academic Editor: Bin Jiang

Copyright (C) 2015 R. Naz and F. M. Mahomed. This is an open access article distributed under the Creative Commons Attribution License, which permits unrestricted use, distribution, and reproduction in any medium, provided the original work is properly cited.

\begin{abstract}
We study a dynamic fourth-order Euler-Bernoulli partial differential equation having a constant elastic modulus and area moment of inertia, a variable lineal mass density $g(x)$, and the applied load denoted by $f(u)$, a function of transverse displacement $u(t, x)$. The complete Lie group classification is obtained for different forms of the variable lineal mass density $g(x)$ and applied load $f(u)$. The equivalence transformations are constructed to simplify the determining equations for the symmetries. The principal algebra is onedimensional and it extends to two- and three-dimensional algebras for an arbitrary applied load, general power-law, exponential, and log type of applied loads for different forms of $g(x)$. For the linear applied load case, we obtain an infinite-dimensional Lie algebra. We recover the Lie symmetry classification results discussed in the literature when $g(x)$ is constant with variable applied load $f(u)$. For the general power-law and exponential case the group invariant solutions are derived. The similarity transformations reduce the fourth-order partial differential equation to a fourth-order ordinary differential equation. For the power-law applied load case a compatible initial-boundary value problem for the clamped and free end beam cases is formulated. We deduce the fourthorder ordinary differential equation with appropriate initial and boundary conditions.
\end{abstract}

\section{Introduction}

Daniel Bernoulli and Leonard Euler developed the theory of the Euler-Bernoulli beam problem. Let $u(t, x)$ be the transverse displacement at time $t$ and position $x$ from one end of the beam taken as the origin, $n(x)$ the flexural rigidity, and $m(x)>0$ the lineal mass. The transverse motion of an unloaded thin beam is represented by the following fourthorder partial differential equation (PDE):

$$
\left(n(x) u_{x x}\right)_{x x}+m(x) u_{t t}=0, \quad t>0,0<x<L .
$$

Euler-Bernoulli beam equation (1) has been frequently studied in the literature. Gottlieb [1] studied the isospectral properties of this equation and its nonhomogeneous variants with $m=1$ and $n=1$. Soh [2] considered the equivalence problem for an Euler-Bernoulli beam utilizing the Lie symmetry approach. Later on Morozov and Soh [3] attempted the problem with the aid of Cartan's equivalence method.
Recently, Ndogmo [4] obtained the complete equivalence transformations of the Euler-Bernoulli equation which were initially considered in the work [3] in terms of some undetermined set of functions. Özkaya and Pakdemirli [5], using the symmetry method, investigated the transverse vibrations of a beam moving with time-dependent axial velocity and obtained approximate solutions for an exponentially decaying and harmonically varying problem.

Now let $E$ be the elastic modulus, let $I$ be the area of inertia, let $\mu$ be the mass per unit length, let $u(t, x)$ be the transverse displacement at time $t$ and position $x$, and let $f$ be the applied load. The transverse motion of a loaded thin elastic beam is governed by the following dynamic beam fourth-order PDE [6]:

$$
\left(E I u_{x x}\right)_{x x}+\mu u_{t t}=f(u),
$$

where the applied load $f$ is a function of $u$. Bokhari et al. [7] studied the following dynamic Euler-beam equation from 
the symmetry viewpoint with $E, I, \mu$ as constants and $f$ dependent on $u$ :

$$
u_{t t}+u_{x x x x}=f(u) .
$$

A complete group classification was obtained for (3). The symmetry reductions were derived to reduce the fourthorder PDE to fourth-order ordinary differential equations (ODEs). For the power-law load function, compatible initialboundary value problems corresponding to clamped end and free end beams were formulated and the reduced fourthorder ODEs were determined. The static beam problem was discussed by Bokhari et al. [8].

The dynamic fourth-order Euler-Bernoulli PDE having a constant elastic modulus and area moment of inertia, a variable lineal mass density $1 / m(x)=g(x)$, and the applied load denoted by $f(u)$, a function of transverse displacement $u(t, x)$, is given by

$$
u_{t t}+g(x) u_{x x x x}=f(u) .
$$

In this paper we study dynamic Euler-Bernoulli beam equation (4) from the symmetry point of view.

We give a complete classification of the Lie symmetries for dynamic Euler-Bernoulli beam equation (4). The principal algebra is one-dimensional and it extends to two- and three-dimensional algebras for an arbitrary applied load, general power-law, exponential, and log type applied loads for different forms of $g(x)$ (see Table 1). For the linear applied load case, we obtain an infinite-dimensional Lie algebra. We recover the Lie symmetry classification results discussed by Bokhari et al. [7] when $g(x)$ is a constant with variable applied load $f(u)$. We derive the group invariant solutions for the general power-law and exponential cases. The fourthorder PDE reduces to a fourth-order ODE with the help of similarity transformations. For the power-law applied load case compatible initial-boundary value problems for the clamped and free end beam cases are formulated. We deduce the corresponding fourth-order ODE with appropriate initial and boundary conditions. We show that the solution fails to satisfy the initial or boundary conditions for the exponential and logarithmic cases.

The paper is organized as follows. In Section 2, the complete Lie point symmetry classification up to equivalence transformations is presented. The nontrivial symmetry reductions and initial-boundary value problems which correspond to clamped and free end beams are discussed in Section 3. The conclusions are summarized in the last section.

\section{Complete Lie Symmetry Classification}

We derive the equivalence transformations which are important for the simplification of the determining equations and for obtaining disjoint classes [9]. Equivalence transformations of the PDE (4) are point transformations in the space of independent and dependent variables of the equation and these point transformations leave invariant family (4). That is, the equivalence transformations transform any equation (4) with arbitrary functions $f$ and $g$ into the same family (4) with, in general, different functions $f$ and $g$. Equivalence transformations of the PDE (4) are easy to obtain although the computations are tedious. These are

$$
\begin{aligned}
& \bar{t}=a_{1} t+a_{2}, \\
& \bar{x}=b_{1} x+b_{2}, \\
& \bar{u}=d_{3} u+d_{4}, \\
& \bar{f}=\frac{d_{3}}{a_{1}^{2}} f, \\
& \bar{g}=\frac{b_{1}^{4}}{a_{1}^{2}} g,
\end{aligned}
$$

where $a_{i}, b_{i}$, and $d_{i}$ are constants and $a_{1} b_{1} d_{3} \neq 0$.

The Lie point symmetry generator

$$
X=\tau(t, x, u) \frac{\partial}{\partial t}+\xi(t, x, u) \frac{\partial}{\partial x}+\eta(t, x, u) \frac{\partial}{\partial u},
$$

of dynamic Euler-Bernoulli equation (4), is derived by solving

$$
\left.X^{[4]}\left[u_{t t}+g(x) u_{x x x x}-f(u)\right]\right|_{(4)}=0,
$$

where $X^{[4]}$ is the fourth prolongation of the operator $X$. The fourth prolongation of the generator $X$ is defined as

$$
X^{[4]}=X+\sum_{s=1}^{4} \zeta_{i_{1}, i_{2}, \ldots, i_{s}} \frac{\partial}{\partial u_{i_{1}, i_{2}, \ldots, i_{s}}},
$$

where $\zeta_{i_{1}, i_{2}, \ldots, i_{s}}$ can be determined from

$$
\begin{gathered}
\zeta_{i}=D_{i}(\eta)-\sum_{j=1}^{2} u_{j} D_{i}\left(\xi^{j}\right), \\
\left(\xi^{1}, \xi^{2}\right)=(\tau, \xi), i=1,2, \\
\zeta_{i_{1}, i_{2}, \ldots, i_{s}}=D_{i_{s}}\left(\zeta_{i_{1}, i_{2}, \ldots, i_{s-1}}\right)-\sum_{j=1}^{2} u_{j i_{1}, i_{2}, \ldots, i_{s-1}} D_{i_{s}}\left(\xi^{j}\right), \\
s=2,3,4, i_{1}, i_{2}, \ldots, i_{s}=1,2
\end{gathered}
$$

in which

$$
D_{i}=\frac{\partial}{\partial x^{i}}+u_{i} \frac{\partial}{\partial u}+u_{i j} \frac{\partial}{\partial u_{j}}+\cdots, \quad\left(x^{1}, x^{2}\right)=(t, x)
$$

is the total derivative operator. Equation (7) is separated according to the derivatives of $u$ and an overdetermined system of partial differential equations for the unknown coefficients $\tau, \xi$, and $\eta$ is obtained. The determining equations finally yield

$$
\begin{aligned}
& \tau=c_{1}+c_{2} t \\
& \xi=c_{3}+c_{4} x+\frac{c_{5}}{2} x^{2} \\
& \eta=\frac{1}{2}\left(3 c_{4}+3 c_{5} x+c_{6}\right) u+a_{2}(t, x)
\end{aligned}
$$


TABLE 1: Complete Lie symmetry classification of beam PDE (4).

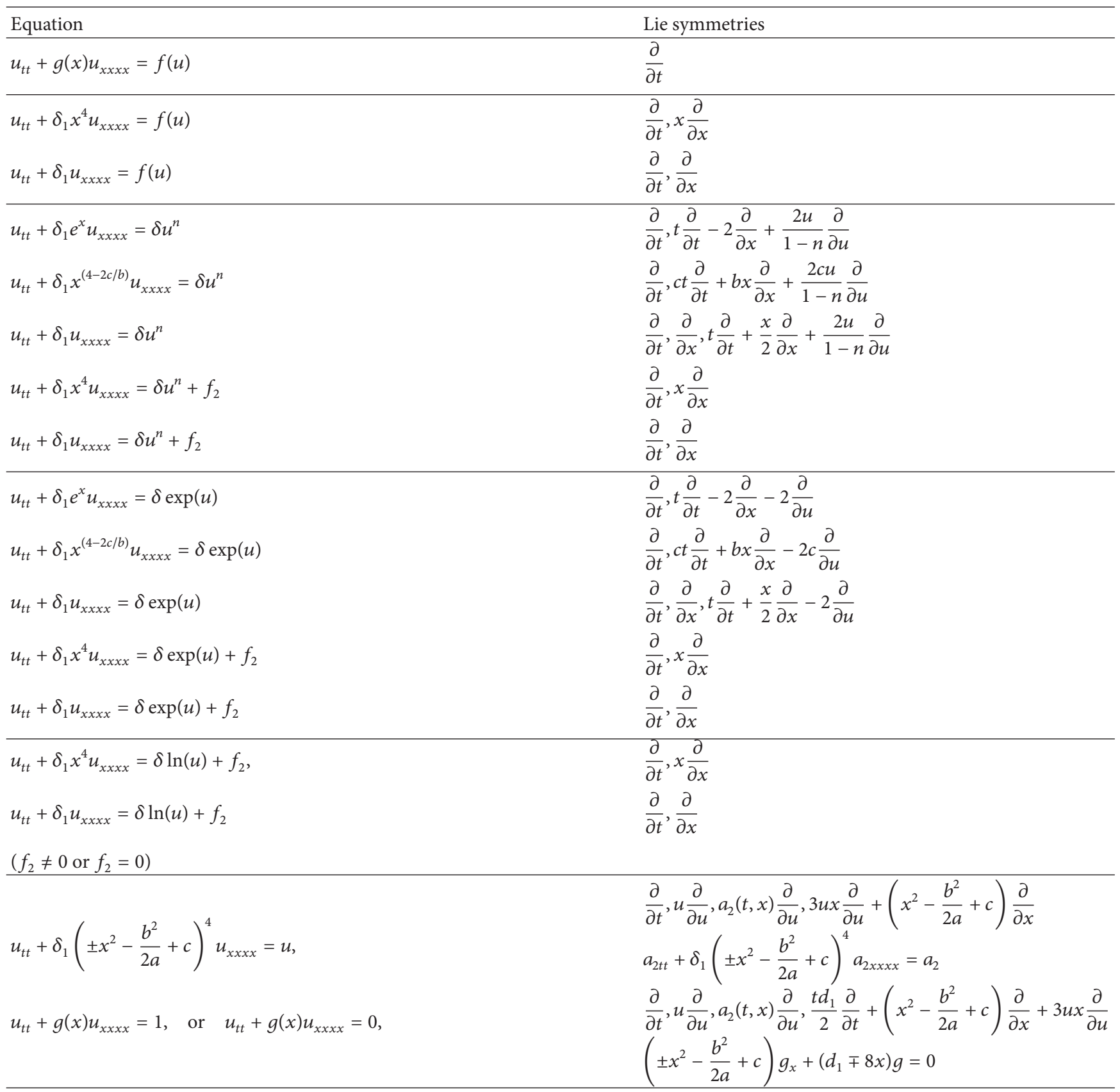

and the following classification equations for $f(u)$ and $g(x)$ :

$$
\begin{array}{r}
\eta_{t t}-\eta f_{u}+g \eta_{x x x x}-2 f \tau_{t}+f \eta_{u}=0 \\
-4 g \xi_{x}+2 \tau_{t} g+\xi g_{x}=0
\end{array}
$$

where $c_{1}, \ldots, c_{6}$ are constants. For the case, $f$ arbitrary in $u$ and $g$ arbitrary in $x$, the only symmetry is

$$
X_{1}=\frac{\partial}{\partial t}
$$

which constitutes the one-dimensional principal algebra of (4). Now we investigate all the possibilities of $f(u)$ and $g(x)$ for which an extension of the principal algebra is possible.
Differentiating classification equation (12) with respect to $u$, we have

$$
\eta f_{u u}+2 \tau_{t} f_{u}=0 .
$$

Further differentiating (15) twice with respect to $u$ yields

$$
\begin{aligned}
\eta f_{u u u}+f_{u u} \eta_{u}+2 \tau_{t} f_{u u} & =0, \\
\eta f_{u u u u}+2 \eta_{u} f_{u u u}+2 \tau_{t} f_{u u u} & =0 .
\end{aligned}
$$

Equation (17) after using $\eta$ from (16) becomes

$$
\left(f_{u} f_{\text {uu }} f_{\text {uuuu }}-2 f_{u} f_{\text {uuu }}^{2}+f_{\text {uu }}^{2} f_{\text {uuu }}\right) \tau_{t}=0 .
$$


Now differentiating (15) with respect to $t$, we have

$$
\eta_{t} f_{u u}=0
$$

We at once look at the possible cases of $f(u)$ from (18) and (19).

Case $1\left(\tau_{t}=0\right)$. If $\tau_{t}=0$ then (15) gives $\eta=0$ and we have

$$
\begin{aligned}
\tau & =c_{1}, \\
\xi & =c_{3}+c_{4} x, \\
\eta & =0,
\end{aligned}
$$

where $f(u)$ is arbitrary and (13) gives $g(x)=\left(c_{3}+c_{4} x\right)^{4}$.

Case $2\left(\tau_{t} \neq 0\right.$ and $\left.f_{u u} \neq 0\right)$. If $\tau_{t} \neq 0$ and $f_{u u} \neq 0$ then (18) yields the following possible forms of $f(u)$ :

(i) $f(u)=f_{1}(a u+b)^{n}+f_{2}$ for $n \neq 0,1, a \neq 0$;

(ii) $f(u)=f_{1} e^{a u}+f_{2}, a \neq 0$;

(iii) $f(u)=f_{1} \ln (a u+b)+f_{2}, a \neq 0$,

where $f_{1}, f_{2}, a, b, n$ are constants and from (19), $\eta_{t}=0$. It is worth mentioning here that the principal algebra extends for these forms of $f(u)$.

After equivalence transformations, the simplified forms for $f(u)$ are

(i) $f(u)=\delta u^{n}+f_{2}$ for $n \neq 0,1$,

(ii) $f(u)=\delta \exp u+f_{2}$,

(iii) $f(u)=\delta \ln u+f_{2}$.

Case 2.1 $\left(f(u)=\delta u^{n}+f_{2}\right)$. Equation (12) for $f(u)=\delta u^{n}+f_{2}$ with $f_{2}=0$ results in the following operators:

$$
\begin{aligned}
\tau & =c_{1}+c_{2} t, \\
\xi & =c_{3}+c_{4} x, \\
\eta & =\frac{2 c_{2}}{1-n} u
\end{aligned}
$$

with $g(x)$ from (13) satisfying

$$
\left(c_{3}+c_{4} x\right) g_{x}+\left(2 c_{2}-4 c_{4}\right) g=0 \text {. }
$$

The solution of $(22)$ is $g(x)=\left(c_{3}+c_{4} x\right)^{4-2 c_{2} / c_{4}}$. When $f_{2} \neq 0$ then $c_{2}=0$ in (21) and (22).

Case 2.2 $\left(f(u)=\delta \exp u+f_{2}\right)$. Equation (12) for $f(u)=$ $\delta \exp u+f_{2}$ with $f_{2}=0$ results in the following operators:

$$
\begin{aligned}
& \tau=c_{1}+c_{2} t, \\
& \xi=c_{3}+c_{4} x, \\
& \eta=-2 c_{2}
\end{aligned}
$$

with $g(x)$ satisfying (22). When $f_{2} \neq 0$ then $c_{2}=0$ in (22) and (23).

Case $2.3\left(f(u)=\delta \ln u+f_{2}\right)$. In this case $\tau_{t}=0$, we have

$$
\begin{aligned}
\tau & =c_{1}, \\
\xi & =c_{3}+c_{4} x \\
\eta & =0
\end{aligned}
$$

with $g(x)$ from (13) satisfying

$$
\left(c_{3}+c_{4} x\right) g_{x}-4 c_{4} g=0 .
$$

The solution of $(25)$ is $g(x)=\left(c_{3}+c_{4} x\right)^{4}$. We deduce the same results for both cases $f_{2}=0$ and $f_{2} \neq 0$.

Case $3\left(f_{u u}=0\right)$. For the case $f(u)=f_{1}+f_{2} u$, the equivalence transformations yield $f(u)=0, f(u)=1$, and $f(u)=$ $\delta u$, where $\delta= \pm 1$.

Case $3.1(f(u)=\delta u)$. Equations (12) and (13) yield

$$
\begin{aligned}
\tau & =c_{1}, \\
\xi & =\frac{c_{5}}{2} x^{2}+c_{4} x+c_{3}, \\
\eta & =\frac{1}{2}\left(c_{6}+3 c_{4}+3 c_{5} x\right) u+a_{2}(t, x),
\end{aligned}
$$

where $g(x)$ and $a_{2}(t, x)$ satisfy

$$
\begin{array}{r}
\left(\frac{c_{5}}{2} x^{2}+c_{4} x+c_{3}\right) g_{x}-\left(4 c_{4}+4 x c_{5}\right) g=0, \\
a_{2 t t}-a_{2}+g(x) a_{2 x x x x}=0 .
\end{array}
$$

Case $3.2(f(u)=1)$. From (12) and (13), we have

$$
\begin{aligned}
\tau & =c_{1}+c_{2} t \\
\xi & =\frac{c_{5}}{2} x^{2}+c_{4} x+c_{3}, \\
\eta & =\frac{1}{2}\left(c_{6}+3 c_{4}+3 c_{5} x\right) u+a_{2}(t, x)
\end{aligned}
$$

with $g(x), a_{2}(t, x)$ satisfying

$$
\begin{array}{r}
\left(\frac{c_{5}}{2} x^{2}+c_{4} x+c_{3}\right) g_{x}+\left(2 c_{2}-4 c_{4}-4 x c_{5}\right) g=0, \\
a_{2 t t}+g(x) a_{2 x x x x}-2 c_{2}+\frac{1}{2}\left(c_{6}+3 c_{4}+3 c_{5} x\right)=0 .
\end{array}
$$

Case $3.3(f(u)=0)$. For this case the symmetry generator is the same as that given in (28) with $g(x)$ satisfying (29) and $a_{2}(t, x)$ :

$$
a_{2 t t}+g(x) a_{2 x x x x}=0 .
$$


Remark 1. The Lie algebras for all the nonlinear cases in Table 1 are easily seen by inspection. For the linear cases, they are infinite-dimensional and well known (see also [9]). Also the solution $g$ of the linear equation in the final linear case of Table 1 is easy to obtain and is not given as we do not use it here.

Now we work out the equivalence transformations for different forms of $g(x)$ arising from Cases 2 and 3. After equivalence transformations $g(x)$ becomes as follows:

(i) $g(x)=g_{1}(a x+b)^{4}, g_{1} \neq 0$, becomes $g=\delta_{1} x^{4}$ for $a \neq 0, \delta_{1}= \pm 1$; if $a=0$, then $g=\delta_{1}$;

(ii) $g(x)=g_{1}(a x+b)^{4-2 c / b}, g_{1} \neq 0, a \neq 0$, becomes $g(x)=$ $\delta_{1} x^{4-2 c / b}, b \neq 0$; if $b=0$, then $g(x)=\delta_{1} \exp x$ with $\delta_{1}= \pm 1$

(iii) if $g$ solves

$$
\left(\frac{a}{2} x^{2}+b x+c\right) g_{x}-(4 b+4 a x) g=0
$$

then after equivalence transformations it reduces to $\left( \pm x^{2}-b^{2} /(2 a)+c\right) g_{x} \mp 8 x g=0$ with solution

$$
g(x)=\delta_{1}\left( \pm x^{2}-\frac{b^{2}}{(2 a)}+c\right)^{4}
$$

(iv) if $g$ satisfies

$$
\left(\frac{a}{2} x^{2}+b x+c\right) g_{x}+(2 d-4 b-4 a x) g=0,
$$

then after equivalence transformations it becomes

$$
\left( \pm x^{2}-\frac{b^{2}}{(2 a)}+c\right) g_{x}+\left(d_{1} \mp 8 x\right) g=0,
$$

where $d_{1} \neq 0$ is arbitrary.

The Lie symmetries for the simplified forms of $f$ and $g$ are presented in Table 1 and all cases discussed in [7] are recovered for $g(x)=\delta_{1}$.

\section{Symmetry Reductions and Boundary Value Problems}

Now we find the symmetry reductions. The initial conditions are

$$
\begin{gathered}
u(0, x)=m(x), \\
u_{t}(0, x)=n(x)
\end{gathered}
$$

The four types of boundary conditions (see [7]) are as follows:

hinged end:

$$
\begin{array}{r}
u(t, 0)=0, \\
u_{x x}(t, 0)=0 ;
\end{array}
$$

clamped end:

$$
\begin{gathered}
u(t, 0)=0 \\
u_{x}(t, 0)=0
\end{gathered}
$$

free end:

$$
\begin{gathered}
u_{x x}(t, 0)=0, \\
u_{x x x}(t, 0)=0 ;
\end{gathered}
$$

sliding end:

$$
\begin{aligned}
u_{x}(t, 0) & =0, \\
u_{x x x}(t, 0) & =0 ;
\end{aligned}
$$

consider the power-law case:

$$
u_{t t}+\delta_{1} x^{(4-2 c / b)} u_{x x x x}=\delta u^{n}
$$

If we take a linear combination of the symmetries $X_{i}$, the initial condition $t=0$ and boundary condition $x=0$ are left invariant only by the scaling symmetry $X_{2}$. The group invariant solution corresponding to $X_{2}$ is

$$
u(t, x)=x^{2 c /(1-n) b} f(z)
$$

where $z=x / t^{b / c}$ is the similarity variable. The substitution of (42) into (41) yields the following ODE:

$$
\begin{aligned}
& \delta_{1} z^{4} f^{\prime \prime \prime \prime}+\frac{8 \delta_{1} c}{(1-n) b} z^{3} f^{\prime \prime \prime}+\frac{b^{2}}{c^{2}} z^{2(1+c / b)} f^{\prime \prime} \\
& +\frac{12 c \delta_{1}}{b(n-1)^{2}}\left(n-1+\frac{2 c}{b}\right) z^{2} f^{\prime \prime}+\frac{b}{c}\left(1+\frac{b}{c}\right) \\
& \cdot z^{1+2 c / b} f^{\prime}-\frac{16 \delta_{1} c}{b(n-1)^{3}}\left(n-1+\frac{2 c}{b}\right)\left(n-1+\frac{c}{b}\right) \\
& \cdot z f^{\prime}+\frac{12 \delta_{1} c}{(n-1)^{4} b}\left(n-1+\frac{2 c}{b}\right)\left(n-1+\frac{c}{b}\right) \\
& \cdot\left(n-1+\frac{2 c}{3 b}\right)=\delta f^{n} .
\end{aligned}
$$

For the clamped end beam, the initial and boundary conditions (36) and (38) yield

$$
\begin{gathered}
f(\infty)=f_{0}, \\
f^{\prime}(\infty)=0, \\
f(0)=0, \\
f^{\prime}(0)=0,
\end{gathered}
$$

where $m(x)=x^{2 c /(1-n) b} f_{0}$. Note that the boundary condition at $x=\infty$ added is due to the solution of the ODE. 
We solve (43) subject to conditions (44) and then (42) forms the solution of the clamped end beam (41).

For the free end beam, the initial and boundary conditions (36) and (39) yield

$$
\begin{gathered}
f(\infty)=f_{0}, \\
f^{\prime}(\infty)=0, \\
f(0)=0, \\
f^{\prime}(0)=0, \\
f^{\prime \prime}(0)=0, \\
f^{\prime \prime \prime}(0)=0 .
\end{gathered}
$$

For the hinged end beam, the ODE (43) should be solved subject to boundary conditions $(44)$ as well as $f^{\prime \prime}(0)=0$. For the case of the sliding end beam one has to solve ODE (43) subject to (45).

For the following power-law case, the PDE is

$$
u_{t t}+\delta_{1} e^{x} u_{x x x x}=\delta u^{n}
$$

and reduction via $X_{2}$ yields the invariant solution

$$
u(t, x)=e^{(1-n) x} f(z), \quad z=x+2 \ln (t) .
$$

Here the second boundary condition $u_{x}(t, 0)=0$ for the clamped end is not satisfied.

An asymptotic solution was found by Bokhari et al. [7] corresponding to the clamped or free end case for the following power-law case

$$
u_{t t}+\delta_{1} u_{x x x x}=\delta u^{n}
$$

For the remaining cases the solution does not satisfy the initial or boundary conditions. We take one example below.

Consider the following exponential case:

$$
u_{t t}+\delta_{1} x^{(4-2 c / b)} u_{x x x x}=\delta e^{u}
$$

The group invariant solution of (49) is of the form

$$
u(t, x)=\ln \left(x^{-2 c / b}\right)+f(z), \quad z=\frac{x}{t^{b / c}} .
$$

The substitution of (50) into (49) yields the following fourthorder ODE:

$$
\begin{gathered}
\delta_{1} z^{4} f^{\prime \prime \prime \prime}+\frac{b^{2}}{c^{2}} z^{2(1+c / b)} f^{\prime \prime}+\frac{b}{c}\left(1+\frac{b}{c}\right) z^{1+2 c / b} f^{\prime} \\
+12 \delta_{1} \frac{c}{b}=\delta e^{f},
\end{gathered}
$$

and this ODE fails to satisfy the boundary conditions at $x=0$.

Similarly for the rest of the cases, the initial or boundary conditions are not satisfied when the load is of exponential or logarithmic form. We have ignored the linear cases as much attention has been already focused on these cases.

\section{Concluding Remarks}

We have performed the complete Lie symmetry classification of the dynamic fourth-order Euler-Bernoulli PDE having a constant elastic modulus and area moment of inertia, a variable lineal mass density $g(x)$, and the applied load denoted by $f(u)$, a function of transverse displacement $u(t, x)$. The equivalence transformations are constructed to simplify the determining equations for the symmetries. The simplified forms of lineal mass density $g(x)$ and applied load $f(u)$ are constructed via equivalence transformations. The principal algebra is one-dimensional for arbitrary $f(u)$ and $g(x)$. The principal algebra is extended to a two- and three-dimensional algebra for arbitrary applied load, general power-law, exponential, and log type of applied loads for different forms of $g(x)$ whereas an infinite-dimensional algebra is obtained for the linear applied load case (see Table 1). We recover the Lie symmetry classification results discussed in the literature when $g(x)$ is constant with variable applied load $f(u)$. The similarity transformations reduce the fourth-order PDE to a fourth-order ODE. Only for one case with the applied load power-law, compatible initial-boundary value problems for the clamped and free end beam cases are formulated. We deduce the fourth-order ODE with appropriate initial and boundary conditions.

\section{Conflict of Interests}

The authors declare that there is no conflict of interests regarding the publication of this paper.

\section{Acknowledgments}

F. M. Mahomed thanks the National Research Foundation (NRF) of South Africa for a research grant that has facilitated this research through the Unique Grant no. 92857, 2014. R. Naz thanks Lahore School of Economics for providing funds to complete this work.

\section{References}

[1] H. P. W. Gottlieb, "Isospectral Euler-Bernoulli beams with continuous density and rigidity functions," Proceedings of the Royal Society of London Series A: Mathematical, Physical and Engineering Sciences, vol. 413, no. 1844, pp. 235-250, 1987.

[2] C. W. Soh, "Euler-Bernoulli beams from a symmetry standpoint-characterization of equivalent equations," Journal of Mathematical Analysis and Applications, vol. 345, no. 1, pp. 387395, 2008.

[3] O. I. Morozov and C. W. Soh, "The equivalence problem for the Euler-Bernoulli beam equation via Cartan's method," Journal of Physics A: Mathematical and Theoretical, vol. 41, no. 13, Article ID 135206, pp. 135-206, 2008.

[4] J. C. Ndogmo, "Equivalence transformations of the Euler-Bernoulli equation," Nonlinear Analysis: Real World Applications, vol. 13, no. 5, pp. 2172-2177, 2012.

[5] E. Özkaya and M. Pakdemirli, "Group-theoretic approach to axially accelerating beam problem," Acta Mechanica, vol. 155, no. 1-2, pp. 111-123, 2002. 
[6] A. E. H. Love, A Treatise on the Mathematical Theory of Elasticity, Dover Publications, New York, NY, USA, 4th edition, 1944.

[7] A. H. Bokhari, F. M. Mahomed, and F. D. Zaman, "Invariant boundary value problems for a fourth-order dynamic EulerBernoulli beam equation," Journal of Mathematical Physics, vol. 53, no. 4, Article ID 043703, 2012.

[8] A. H. Bokhari, F. M. Mahomed, and F. D. Zaman, "Symmetries and integrability of a fourth-order Euler-Bernoulli beam equation," Journal of Mathematical Physics, vol. 51, no. 5, Article ID 053517, 2010.

[9] N. H. Ibragimov, Ed., CRC Handbook of Lie Group Analysis of Differential Equations, vol. 1-3, Chemical Rubber Company, Boca Raton, Fla, USA, 1994-1996. 


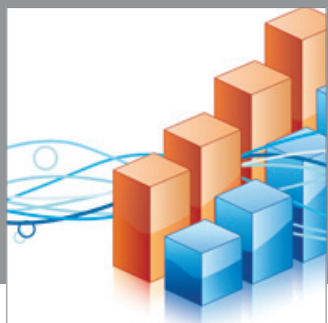

Advances in

Operations Research

mansans

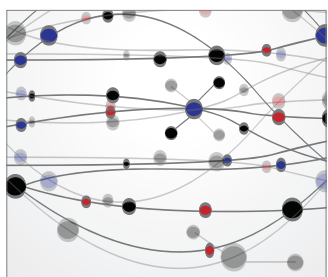

The Scientific World Journal
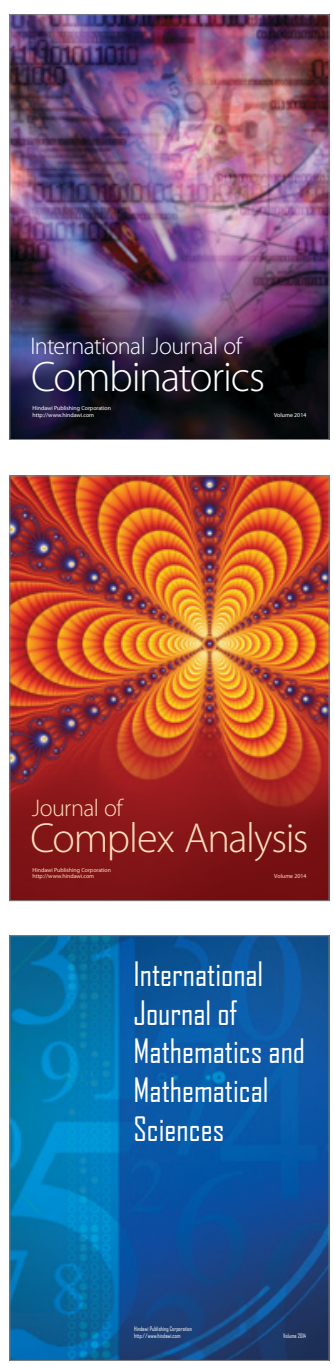
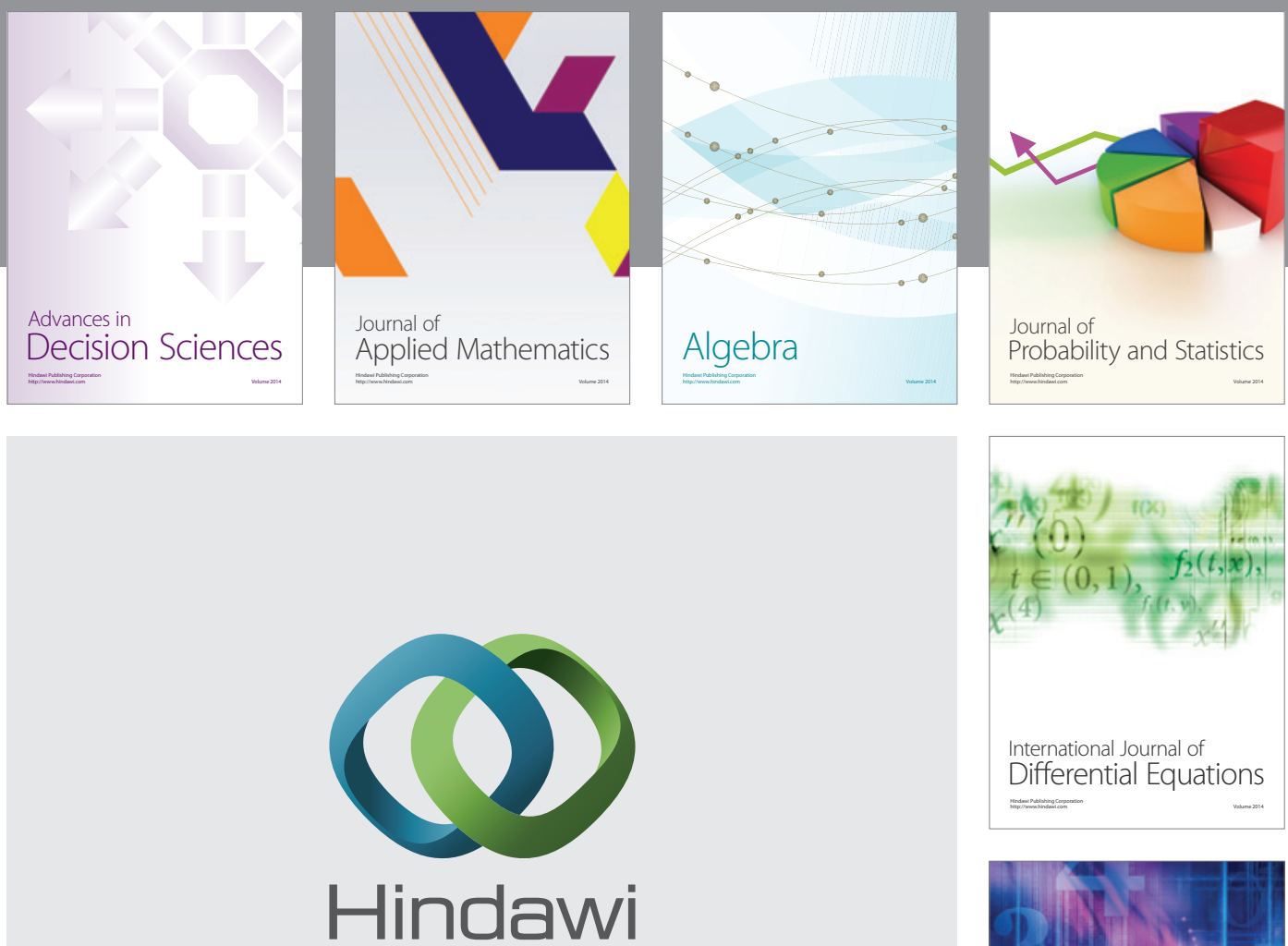

Submit your manuscripts at http://www.hindawi.com
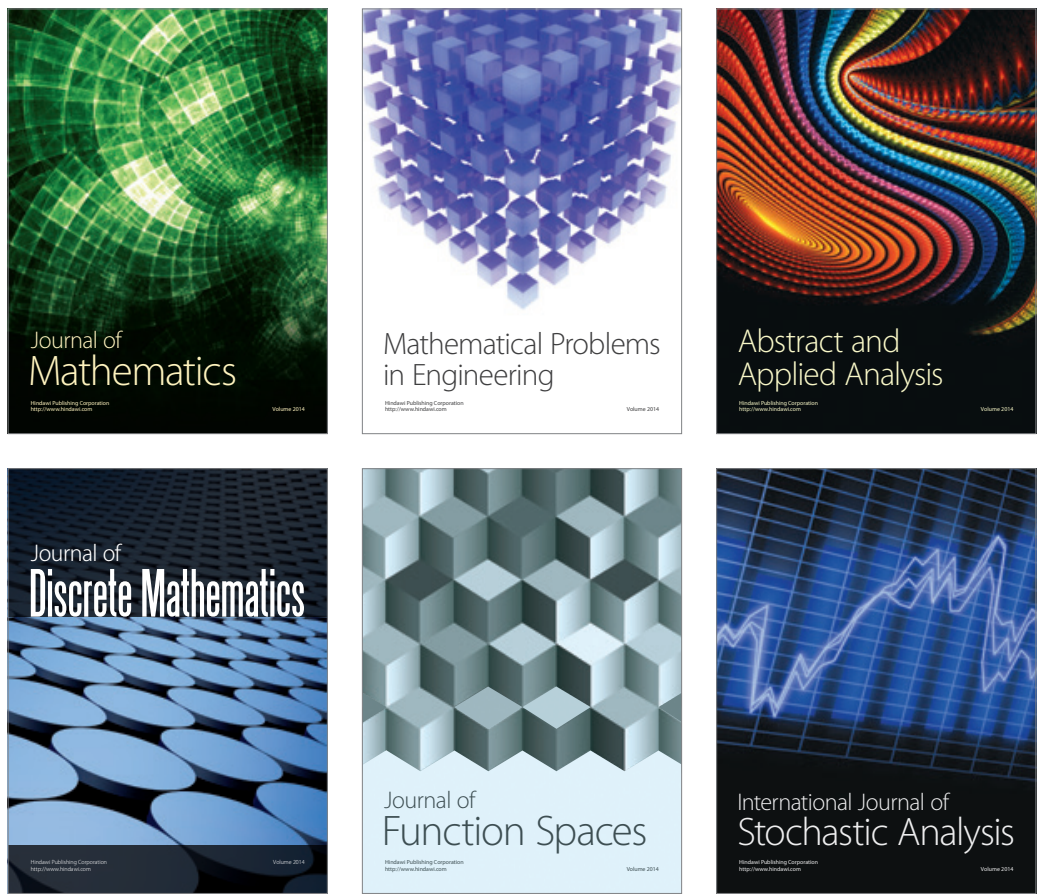

Journal of

Function Spaces

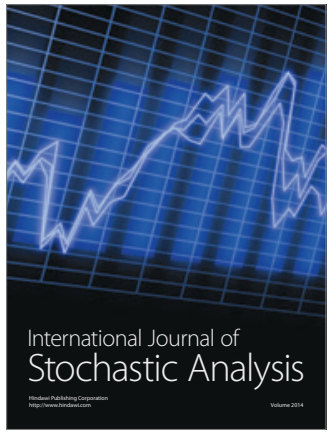

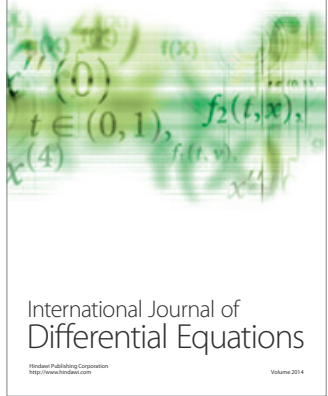
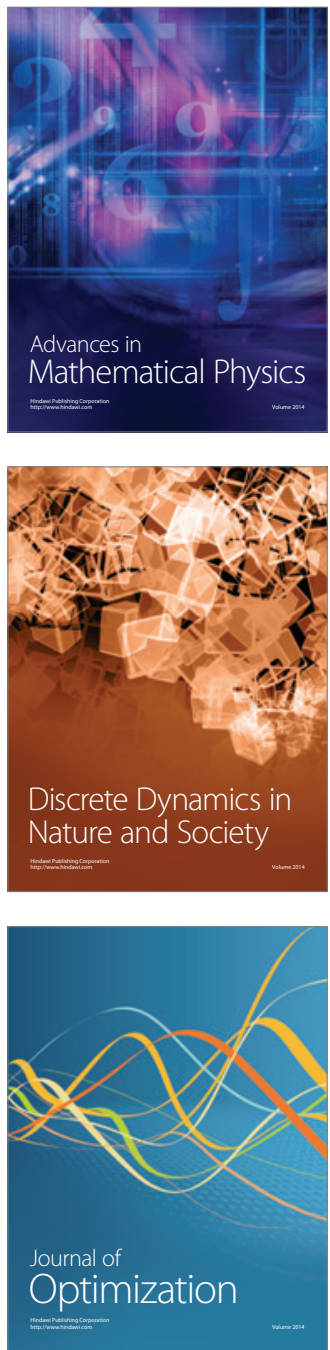